\title{
TRATAMENTO FARMACOLÓGICO DAS DOENÇAS REUMÁTICAS
}

\author{
PHARMACOLOGIC THERAPY OF RHEUMATIC DISEASES
}

Flávio C Petean ${ }^{1}$, Júlio C Voltarelli², Eduardo A. Donadi ${ }^{2}$, Paulo Lousada $\mathrm{Jr}^{1}$, Ivan F. de Carvalho ${ }^{2}$

\begin{abstract}
${ }^{1}$ Médicos-Assistentes, ${ }^{2}$ Docentes da Divisão de Imunologia Clínica do Departamento de Clínica Médica da Faculdade de Medicina de Ribeirão Preto da Universidade de São Paulo.

CORRESPONDÊNCIA: Divisão de Imunologia Clínica - Departamento de Clínica Médica-HCFMRP-USP - Campus Universitário - CEP: 14048900 - Ribeirão Preto - SP.
\end{abstract}

PETEAN FC et al. Tratamento farmacológico das doenças reumáticas. Medicina, Ribeirão Preto, 30: 522-531, out./dez. 1997.

RESUMO: Apresenta-se, nesta revisão, a terapêutica farmacológica atual das doenças reumáticas de adultos, do modo como é conduzida na Divisão de Imunologia Clínica do Hospital das Clínicas da Faculdade de Medicina de Ribeirão Preto, da Universidade de São Paulo. Enfatizam-se a seleção de drogas, as respostas clínicas e o monitoramento terapêutico das principais doenças reumáticas. Entre estas, incluem-se tanto as de patogênese auto-imune (artrite reumatóide, lúpus eritematoso sistêmico, febre reumática, espondilartropatias, esclerose sistêmica, dermatopolimiosite, vasculites), como as não-imunológicas (gota, pseudogota, osteoartrose, osteoporose e fibromialgia).

UNITERMOS: Doenças Reumáticas. Farmacologia. Antiinflamatórios. Imunossupressores.

Discute-se, nesta revisão, a abordagem farmacoterapêutica das principais doenças reumáticas de adultos, a maioria das quais, mas não todas, possui uma patogênese auto-imune. Esta abordagem reflete a experiência acumulada pela Divisão de Imunologia Clínica do Departamento de Clínica Médica da FMRPUSP, em 22 anos de atividades. Pela extensão do tema, não foi possível discutir as características farmacológicas das drogas citadas, o que foi feito em artigo precedente ${ }^{(1)}$, nem as novas drogas que estão, atualmente, sendo testadas em estudos clínicos. Pelo mesmo motivo, não foram consideradas, em profundidade, importantes modalidades terapêuticas, não farmacológicas, para doenças reumáticas (fisioterapia, psicoterapia, nutrição, terapias alternativas, etc).

\section{ARTRITE REUMATÓIDE}

Na última década, houve importante mudança na terapêutica da artrite reumatóide (AR), reservando-se a clássica abordagem piramidal para os casos mais leves e de evolução mais benigna. Nessa abordagem, introduzem-se, progressivamente, drogas mais potentes e mais tóxicas, à medida em que a doença se agrava. $O$ consenso atual da literatura, entretanto, ainda é o controle inicial da inflamação com antiinflamatórios não-esteroidais (AINE) (drogas de primeira linha), com uma aplicação mais precoce de agentes imunossupressores ou imunomoduladores (drogas remissivas ou de segunda linha) (Tabela I). A maioria dessas drogas demora vários 
meses (três a seis) para mostrar seus efeitos benéficos, necessitando de ser combinadas com antiinflamatórios de ação mais rápida. Entre as drogas remissivas, aparentemente, o metotrexato tem uma ação mais precoce, dentro de três a seis semanas de uso.

Tabela I - Drogas utilizadas no tratamento da artrite reumatóide*

$1^{\text {a linha }}$

- Antiinflamatórios não-esteroidais

$2^{\mathrm{a}}$ linha

- Antimaláricos

- Metotrexato

- Sulfassalazina

- Sais de ouro

- D-penicilamina

- Ciclosporina

- Azatioprina

- Ciclofosfamida

- Clorambucil

Corticoesteróides

- Dose baixa via oral

- Pulsos endovenosos

- Injeção intra-articular

Agentes em investigação

${ }^{\star}$ Adaptada da ref. (4)

Os corticoesteróides, apesar de sua grande potência antiinflamatória e de suas propriedades imunossupressoras, são reservados para os casos refratários, devido à gravidade dos seus efeitos colaterais, principalmente associados à automedicação. Na ausência de efeito dos AINE, podem, entretanto, ser utilizados, em doses baixas (até $15 \mathrm{mg} / \mathrm{d}$ de prednisona), até que as drogas remissivas comecem a agir. Podem também ser injetados no interior das articulações, quando uma ou poucas delas resistem à terapêutica sistêmica. Pulsos com megadoses de corticoesteróides (500 a 1000 mg de metilprednisolona EV, em três dias consecutivos) são raramente necessários para controle de doença, a não ser em casos refratários ou com envolvimento de órgãos nobres, como a vasculite reumatóide.

Assim, se o paciente apresentar menos de quatorze articulações individuais acometidas, ausência de fator reumatóide e de marcadores imunogenéticos de suscetibilidade do sistema HLA e nenhuma ou pouca lise óssea radiológica, pode-se usar uma das seguintes drogas de segunda linha:

a) antimaláricos: difosfato de cloroquina $(250 \mathrm{mg} / \mathrm{d})$ ou sulfato de hidroxicloroquina $(400 \mathrm{mg} / \mathrm{d}$ );

b) sais de ouro: por via oral (auranofin- $3 \mathrm{mg} 2 \mathrm{x} / \mathrm{d}$ ) ou intramuscular (aurotioglicose ou aurotiomalato$50 \mathrm{mg} / \mathrm{semana}$ até atingir uma dose acumulada de 1 a 2 g, espaçando-se, então, os intervalos das aplicações até uma vez ao mês). A resposta a essas drogas diminui com a idade, não se usando em pacientes acima de quarenta anos;

c) metotrexato: $(7,5$ a $25 \mathrm{mg} / \mathrm{semana} \mathrm{VO}$, divididos em duas a três vezes ou IM, em aplicação única).

Se, por outro lado, a doença se revelar mais agressiva, com acometimento de quatorze ou mais articulações e, particularmente, das metatarsofalangeanas, com a presença de fator reumatóide, nódulos reumatóides e outras manifestações extra-articulares e de antígenos HLA predisponentes (HLA-DRB1 0101, 0401, 0404) utilizam-se, além dos antiinflamatórios, combinações de drogas de segunda linha. $O$ esquema poliquimioterápico mais empregado em nosso meio é a associação de antimaláricos com metotrexato, pelo seu baixo custo, baixa incidência de efeitos colaterais e pela aparente proteção conferida pela cloroquina contra a toxicidade hepática do metotrexato.

Quanto maior o número de índices de mau prognóstico, mais drogas podem ser utilizadas, podendo-se acrescentar, por exemplo, sulfassalazina $(500 \mathrm{mg} 1 \mathrm{x} / \mathrm{d}$, aumentando-se, semanalmente, até atingir 2-3 g/d), D-penicilamina $(250 \mathrm{mg} / \mathrm{d}$, aumentando-se $125 \mathrm{a} 250 \mathrm{mg}$ a cada 4-8 semanas, até atingir 750-1000 mg/d) e/ou sais de ouro ao esquema básico de cloroquina + metotrexato. Ocorrendo falha terapêutica ou progressão da doença (radiológica ou extra-articular), mesmo com melhora da inflamação articular, pode-se acrescentar um dos imunossupressores clássicos: azatioprina ( 1,5 a $2,5 \mathrm{mg} / \mathrm{kg} / \mathrm{d})$, clorambucil $(0,1$ a $0,2 \mathrm{mg} / \mathrm{kg} / \mathrm{d})$, ciclofosfamida (1 a $2 \mathrm{mg} / \mathrm{kg} / \mathrm{d}$ ) ou ciclosporina (2,5 a 5 $\mathrm{mg} / \mathrm{kg} / \mathrm{d}$ ), todos por via oral. Conforme a doença vai sendo controlada, o número de drogas e suas doses vão sendo paulatinamente reduzidos.

O seguimento do paciente deve ser realizado em intervalos mais curtos no início do tratamento, para detectar qualquer reação adversa que venha a ocorrer. A poliquimioterapia anti-reumática não parece ter aumentado o risco dessas reações em relação ao observado com o uso isolado das drogas. Entretanto, em 
um dos primeiros estudos realizados, nesta linha, por McCarty, em 1982, a ciclofosfamida, combinada à azatioprina e à hidroxicloroquina, induziu neoplasias em vários pacientes e foi substituída pelo metotrexato. Os principais efeitos adversos, que precisam ser monitorizados laboratorialmente na terapêutica farmacológica das doenças reumáticas, ao lado das doses habituais das drogas anti-reumáticas, estão indicados na Tabela II.

O efeito mielotóxico das drogas citotóxicas usadas como imunossupressores (ciclofofamida, clorambucil, azatioprina, metotrexato e outras) é monitorizado por exames hematológicos convencionais, raramente sendo necessárias aspirações de medula óssea. As doses dessas drogas são ajustadas para se manter uma leucopenia leve (leucócitos totais em torno de 3000/ $\mu 1$ e neutrófilos em torno de $1500 / \mu 1)$. Em nosso serviço, os pulsos de ciclofosfamida iniciam-se com doses de $500 \mathrm{mg} / \mathrm{m}^{2}$, aumentando-se, subsequientemente, $20 \%$ da dose a cada ciclo, até se atingir o efeito leucopenizante desejado, o que requer uma dose em torno de 800 a $1200 \mathrm{mg} / \mathrm{m}^{2}$.

\section{LÚPUS ERITEMATOSO SISTÊMICO}

A terapêutica do lúpus eritematoso sistêmico (LES) é tão diversificada quanto as próprias mani- festações clínicas da doença, que podem ocorrer em praticamente todos os sistemas orgânicos. A identificação do grau e extensão deste acometimento é essencial para o planejamento terapêutico do LES, que é apresentado abaixo, dividido por sistema afetado.

\subsection{Manifestações cutâneas}

Antes de receber qualquer medicação, o paciente deve ser orientado a evitar exposição prolongada ao sol, sendo sempre recomendado o uso de bloqueadores solares; mesmo para exposições breves, deve ser usado filtro solar de potência maior do que 30 . A droga de escolha para o tratamento sistêmico de lesões cutâneas é um antimalárico (Tabela II). Nos poucos casos refratários, pode-se usar corticoesteróides sistêmicos (prednisona $0,5 \mathrm{mg} / \mathrm{kg} / \mathrm{d}$ ) ou tópicos, para lesões localizadas. As preparações tópicas de hidrocortisona são preferidas e as fluoretadas (dexametasona, betametasona e triancinolona) não devem ser usadas por mais de duas semanas.

Outras drogas utilizadas para manifestações cutâneas são: dapsona $(100 \mathrm{mg} / \mathrm{d})$, principalmente para lesões bolhosas, talidomida (100 mg/d), cujos efeitos teratogênicos limitam seu uso em mulheres em idade fértil, metotrexato (mesma dose da AR) e clofazimina (droga antihansênica, ainda não utilizada em nosso serviço).

\begin{tabular}{|c|c|c|c|c|}
\hline Droga & Dose habitual & Principais efeitos adversos & Controle laboratorial & Intervalo \\
\hline Antimaláricos & $\begin{array}{l}250 \mathrm{mg} / \mathrm{d} \text { DFCQ } \\
400 \mathrm{mg} / \mathrm{d} \mathrm{HCQ}\end{array}$ & Degeneração macular & Fundoscopia & 6 meses \\
\hline Sais de ouro & $\begin{array}{l}50 \mathrm{mg} / \mathrm{sem} \text { AuTG } \\
6 \mathrm{mg} / \mathrm{d} \text { AuNF }\end{array}$ & $\begin{array}{l}\text { Nefrite, citopenias hematológicas, } \\
\text { anemia aplástica, dermatite }\end{array}$ & $\begin{array}{l}\text { Urina rotina, } \\
\text { hematológico }\end{array}$ & $\begin{array}{l}2 \text { sem } \Rightarrow \\
\text { mensal }\end{array}$ \\
\hline D-penicilamina & $750 \mathrm{mg} / \mathrm{d}$ & Idem & Idem & Idem \\
\hline Sulfassalazina & $2 \mathrm{~g} / \mathrm{d}$ & $\begin{array}{l}\text { Dispepsia, citopenias hemato- } \\
\text { lógicas, anemia hemolítica }\end{array}$ & Hematológico & Idem \\
\hline Metotrexato & $7,5-15 \mathrm{mg} / \mathrm{sem}$ & Fibrose ou cirrose hepática & Enzimas hepáticas & $6-8$ sem \\
\hline Ciclosporina & $3 \mathrm{mg} / \mathrm{kg} / \mathrm{d}$ VO & Insuficiência renal & Creatinina & mensal \\
\hline Ciclofosfamida & $\begin{array}{l}1-2 \mathrm{mg} / \mathrm{kg} / \mathrm{d} \text { VO } \\
0,5-1 \mathrm{~g} / \mathrm{m}^{2} \mathrm{EV}\end{array}$ & Citopenias hematológicas & $\begin{array}{l}\text { Hematológico } \\
\text { Idem }\end{array}$ & $\begin{array}{l}\text { mensal } \\
+10-14 \mathrm{~d}\end{array}$ \\
\hline Azatioprina & $1,25-2,5 \mathrm{mg} / \mathrm{kg} / \mathrm{d}$ & Idem & Idem & Idem \\
\hline Clorambucil & $0,1-0,2 \mathrm{mg} / \mathrm{kg} / \mathrm{d}$ & Idem & Idem & Idem \\
\hline Prednisona & $10 \mathrm{mg} / \mathrm{d}-2 \mathrm{mg} / \mathrm{kg} / \mathrm{d}$ & $\begin{array}{l}\text { Osteoporose } \\
\text { Hiperglicemia, hipocalemia }\end{array}$ & $\begin{array}{l}\text { Densiometria óssea } \\
\text { Glicemia, eletrólitos }\end{array}$ & $\begin{array}{l}1 \text { ano } \\
1 \text { mês }\end{array}$ \\
\hline M-prednisolona & $1 \mathrm{~g} / \mathrm{d} \times 3$ & $\begin{array}{l}\text { Hiperglicemia, hipocalemia, } \\
\text { retenção hídrica, } \\
\text { hipertensão arterial }\end{array}$ & Glicemia, eletrólitos & Diário \\
\hline
\end{tabular}

DFCQ: difosfato de cloroquina, HCQ: hidroxicloroquina, AuTG: aurotioglicose, AuNF: auranofin 


\subsection{Manifestações articulares}

Como o LES, via de regra, não produz seqüelas articulares graves, os sintomas articulares podem ser costumeiramente controlados com AINE, baixas doses de corticoesteróides ou cloroquina. Nas situações excepcionais, que exijam drogas imunossupressoras, pode-se usar metotrexato ou azatioprina.

\subsection{Serosites}

Classicamente, as serosites regridem com o uso de indometacina (75 a $150 \mathrm{mg} / \mathrm{d}$ ); quando isto não ocorre, recorre-se aos corticoesteróides orais (prednisona 0,5 a $1,0 \mathrm{mg} / \mathrm{kg} / \mathrm{d}$ ). Nos grandes derrames pleurais ou pericárdicos, com repercussões funcionais (restrição respiratória ou tamponamento cardíaco), temos usado pulsos de metilprednisolona endovenosa (Tabela II).

\subsection{Manifestações hematológicas}

As citopenias auto-imunes do LES (anemia hemolítica, trombocitopenia e leucopenia) costumam responder a doses altas de corticoesteróides VO (prednisona $1,0-2,0 \mathrm{mg} / \mathrm{kg} / \mathrm{d}$ ). Nos casos refratários ou com efeitos colaterais exagerados, temos tido sucesso com o uso de imunoglobulina endovenosa (1 a $2 \mathrm{~g} / \mathrm{kg}$ mês). Além de ter um custo bastante elevado e poder causar insuficiência renal, aparentemente, este tratamento é mais eficiente para a trombocitopenia do que para as outras citopenias. Quando não for possível utilizá-lo, pode-se recorrer a drogas citotóxicas (azatioprina, ciclofosfamida, vincristina ou clorambucil) ou, excepcionalmente, à esplenectomia.

\subsection{Manifestações neuropsiquiátricas}

Além da terapêutica sintomática para as várias síndromes neuropsiquiátricas (anticonvulsivantes, neurolépticos, antidepressivos, etc), a supressão da atividade da doença pode ser obtida com corticoesteróides (VO ou em pulsos, para os casos mais gra- ves) ou drogas citotóxicas (azatioprina ou ciclofosfamida). Em nossa experiência, temos usado com sucesso pulsos de ciclofosfamida $\left(0,5\right.$ a $1,0 \mathrm{~g} / \mathrm{m}^{2}$ mensal) em casos graves de cerebrite lúpica, que podem ocorrer na ausência de outras manifestações da doença.

\subsection{Manifestações vasculares}

A vasculite lúpica é muito comum e, na dependência de seu órgão-alvo, pode ser bastante deletéria ao paciente. Sendo assim, utilizamos a prednisona $(1 \mathrm{mg}$ / $\mathrm{kg} / \mathrm{d}$ ) nos casos de risco menor (vasculite cutânea) e outros imunossupressores nos de maior risco (vasculite intestinal ou cerebral, por exemplo). A droga mais utilizada é a ciclofosfamida na forma de pulso (vide Tabela II). Se, por outro lado, as manifestações vasculares forem secundárias à síndrome do anticorpo antofosfolípide, os imunossupressores são pouco eficientes e o tratamento é dirigido aos fenômenos trombóticos: heparina (convencional $5000 \mathrm{U} \mathrm{SC}$ 12/12 h ou de baixo peso molecular $40 \mathrm{mg} / \mathrm{d} \mathrm{SC}$ ) na fase aguda e anticoagulantes orais para manutenção, ajustando-se as doses para que o "International Normatizing Ratio" (INR) do Tempo de Protrombina fique entre 3,5 e 4,5. Nas vasculites cutâneas, usam-se também drogas vasodilatadoras (nifedipina, pentoxifilina e outras). Nosso serviço tem grande experiência com o emprego de pulsos endovenosos de xilocaína (ver item 5).

\subsection{Manifestações renais}

O quadro renal é o mais temido no paciente lúpico, devido a sua alta freqüência e gravidade, levando, muitas vezes, à insuficiência renal crônica e, mesmo, à morte. $\mathrm{O}$ tratamento da nefrite lúpica vai depender de vários fatores, como a idade, sexo, complicações clínicas, alterações laboratoriais, fatores logísticos e, principalmente, do quadro histopatológico renal (Tabela III).

Tabela III - Terapêutica da glomerulonefrite (GN) lúpica

\begin{tabular}{|c|c|c|}
\hline \multirow{2}{*}{$\begin{array}{l}\text { Classificação } \\
\text { histopatológica }\end{array}$} & \multicolumn{2}{|c|}{ Esquema terapêutico } \\
\hline & $1^{\mathrm{a}}$ escolha & $2^{\mathrm{a}}$ escolha \\
\hline GN mesangial & Prednisona $1 \mathrm{mg} / \mathrm{kg} / \mathrm{d}$ VO & Metilprednisolona $1 \mathrm{~g} / \mathrm{d}$ x3/mês EV \\
\hline GN proliferativa focal & Ciclofosfamida 0,5-1,0 g/m2 EV & Azatioprina $1,5-2,5 \mathrm{mg} / \mathrm{kg} / \mathrm{d}$ \\
\hline GN proliferativa difusa & Idem & $\begin{array}{l}\text { Azatioprina 1,5-2,5 mg/kg/d VO + } \\
\text { Ciclofosfamida 1-2 mg/kg/d VO }\end{array}$ \\
\hline GN membranosa & Metilprednisolona $1 \mathrm{~g} / \mathrm{d}$ x3/mês EV & Azatioprina 1,5-2,5 mg/kg/d VO \\
\hline Mistas & $\begin{array}{l}\text { Usar o esquema dirigido ao tipo de } \\
\text { pior prognóstico }\end{array}$ & \\
\hline
\end{tabular}


Entretanto, algumas medidas gerais são aplicáveis a todos os pacientes: redução da ingesta de sal, gordura e proteínas, na ocorrência, respectivamente, de hipertensão arterial, hiperlipidemia e retenção nitrogenada. Nesse caso, o uso de diuréticos de alça, como a furosemida no controle de edema, deve ser cauteloso. Do mesmo modo, ocorrendo crises de atividade inflamatória lúpica em outros setores do organismo, o emprego de AINE também deve ser cuidadoso, pelo risco de toxicidade renal.

\subsubsection{Glomerulonefrite mesangial}

Em muitos serviços, não se trata este tipo de nefropatia, devido à sua benignidade, na maioria dos casos. Em nosso serviço, entretanto, optamos pelo tratamento com prednisona VO $(1 \mathrm{mg} / \mathrm{kg} / \mathrm{d})$ ou por pulsos de metilprednisolona nos casos refratários.

\subsubsection{Glomerulonefrite proliferativa focal}

Não há consenso sobre o melhor tipo de tratamento para este tipo de nefrite. Em nosso serviço, tratamos, de preferência, com pulsos de ciclofosfamida (mensal, por seis meses, e trimestral por mais três anos), pelo potencial de transformação na forma difusa. Nos casos de boa evolução e de menor gravidade, pode-se usar azatioprina pelo mesmo período.

\subsubsection{Glomerulonefrite proliferativa difusa}

Esta é a mais grave das nefropatias lúpicas, levando, freqüentemente, ao comprometimento irreversível da função renal. É, também, a única forma em que se comprovou o efeito benéfico dos pulsos de ciclofosfamida na progressão da doença. Em nosso serviço, usamos pulsos mensais por seis a doze meses, e a cada dois ou três meses por quatro anos. Quando o paciente não responde ou, por qualquer motivo, não consegue completar o tratamento, pode-se usar uma combinação de azatioprina (1,5 a $2,5 \mathrm{mg} / \mathrm{kg} / \mathrm{d}$ ) com ciclofosfamida (1 a $2 \mathrm{mg} / \mathrm{kg} / \mathrm{d}$ ).

\subsubsection{Glomerulonefrite membranosa}

Há vários esquemas terapêuticos para este tipo de nefrite, mas os mais utilizados são os pulsos mensais de metilprednisolona e azatioprina oral.

\subsubsection{Glomerulonefrites mistas}

Usa-se terapêutica dirigida ao tipo histopatológico de maior gravidade.

Para finalizar, dois aspectos pertinentes à terapêutica clínica do LES merecem comentários. Em primeiro lugar, como os corticoesteróides são as drogas mais utilizadas nos vários esquemas terapêuticos, uma vez obtida a resposta esperada, ou na vigência de reações adversas inaceitáveis, sua dose deve ser reduzida lentamente (5-10\%/semana), para evitar tanto a "síndrome de abstinência" como as conseqüências da supressão do eixo hipotálamo-hipófisário adrenal. Em segundo lugar, apesar da controvérsia quanto à contra-indicação da gravidez nas várias formas de LES, parece não haver dúvidas quanto aos riscos da gravidez na nefrite lúpica ativa.

\section{ESPONDILOARTROPATIAS}

Este grupo nosológico inclui a espondilite anquilosante, a síndrome de Reiter, a espondilite psoriásica e aquelas associadas a doenças inflamatórias intestinais. De uma maneira geral, o planejamento terapêutico dessas doenças visa o controle das manifestações inflamatórias, a preservação da mobilidade da coluna vertebral através de um vigoroso programa fisioterápico e o controle das manifestações extra-articulares. Entretanto, no caso específico da forma aguda da síndrome de Reiter, deve-se adicionar um esquema antiinfeccioso para eliminar possíveis agentes desencadeantes da doença (Chlamydia e bactérias intestinais).

Do ponto de vista farmacológico, o esquema terapêutico é muito semelhante para todas as artropatias. A droga de escolha para o início do tratamento é a indometacina, na dose de 75 a $200 \mathrm{mg} / \mathrm{d}$; para pacientes com acentuada intolerância gástrica ou muitos sintomas noturnos e matinais, pode-se usar a droga na forma de supositórios (100 mg). Outros AINE também podem ser empregados, sendo as espondiloartropatias a única indicação para o uso de fenilbutazona (dose inicial de 400-600 mg e manutenção de $300 \mathrm{mg} / \mathrm{d}$ ).

$\mathrm{Na}$ ausência de resposta aos AINE e na vigência de doença progressiva, usam-se drogas remissivas de ação lenta (veja drogas de segunda linha da AR- Tabela I). O esquema mais utilizado emprega sulfassalazina ( 2 a $4 \mathrm{~g} / \mathrm{d})$, produzindo, na maioria dos casos, controle do processo inflamatório, da rigidez de repouso e, possivelmente, retardo na progressão da anquilose da coluna vertebral. Nosso serviço tem também atingido esses objetivos com o uso de metotrexato, em uma dose média semanal de 10 a 15 $\mathbf{m g} / \mathbf{s e m}$. Imunossupressores clássicos (azatioprina, ciclofofamida, clorambucil e ciclosporina) constituem alternativa terapêutica para os casos refratários.

Para crises agudas da doença, resistentes aos AINE, pode-se empregar doses altas de corticoeste- 
róides (VO ou EV). Este esquema está contra-indicado nas espondiloartropatias associadas ao HIV, pelo risco de imunossupressão adicional, e à artrite psoriásica, pela possibilidade de exacerbação das lesões cutâneas, quando se reduz a dose dos corticoesteróides.

\section{DERMATOMIOSITE E POLIMIOSITE}

$\mathrm{O}$ tratamento farmacológico das miopatias inflamatórias tem evoluído consideravelmente, nos últimos anos. A primeira escolha ainda é a prednisona (1 a $2 \mathrm{mg} / \mathrm{kg} / \mathrm{d}$ ) até a normalização dos níveis séricos das enzimas musculares. A redução da dose deve ser bastante lenta (1/4 da dose inicial por mês) para se evitar reativação da doença. Ao se atingir a dose de $20 \mathrm{mg} / \mathrm{d}$, esta deve ser mantida por seis a doze meses, de preferência em dias alternados, quando, então, se reduz $5 \mathrm{mg} / \mathrm{mês}$ até a dose de $5 \mathrm{mg} /$ dia.

Há, porém, um grupo de pacientes com manifestações mais graves (acometimento da musculatura da fala, deglutição, respiração e/ou coração, presença de calcinose, vasculite ou pneumopatia) em que está indicado o uso de imunossupressores (metotrexato, azatioprina, ciclofosfamida, ciclosporina) associados aos corticoesteróides. Esses imunossupressores devem ser utilizados, também, nos casos refratários à corticoterapia ou com efeitos colaterais intoleráveis.

Recentemente, tem sido relatado efeito benéfico da infusão de imunoglobulina endovenosa $(2 \mathrm{~g} / \mathrm{kg} /$ mês) em casos resistentes ao tratamento clássico. Em nosso serviço, temos confirmado esta observação, mas o seu uso é limitado pelo alto custo.

As lesões cutâneas da dermatopolimiosite que não responderam à corticoterapia sistêmica podem ser tratadas com antimaláricos. Por outro lado, a calcinose difusa pode ser tratada com etidronato de sódio (10 mg/d por três meses), repetindo-se o esquema ciclicamente.

\section{ESCLEROSE SISTÊMICA}

O tratamento da esclerose sistêmica (ES) e de suas variantes é pouco gratificante, dada sua má resposta terapêutica às drogas existentes. Por essa razão, o esclarecimento do paciente quanto ao prognóstico da doença assume fundamental importância.

O objetivo principal da terapêutica da ES é evitar ou retardar o processo de fibrose nos órgãos acometidos pela doença. Para isto, são utilizadas a Dpenicilamina $(0,25$ a $1,0 \mathrm{~g} / \mathrm{d})$ e a colchicina $(10 \mathrm{mg} /$ sem), as quais, entretanto, são pouco toleradas e, em geral, pouco eficientes. Uma vez estabelecida a fibrose, o processo de reversão é bastante longo e as drogas devem ser usadas por vários anos.

$O$ principal esquema terapêutico utilizado em nosso serviço é constituído de infusões endovenosas de xilocaína $(400 \mathrm{mg} / \mathrm{d}$ por cinco dias consecutivos, repetidos a cada dois meses). Com esse esquema, observamos, ao longo de vários anos, benefícios nítidos sobre a esclerose cutânea e sobre as alterações vasculares da doença e, curiosamente, uma incidência muito baixa da "crise esclerodérmica renal" que, na literatura, ocorre em aproximadamente $20 \%$ dos pacientes com ES.

Doses baixas de prednisona (até $15 \mathrm{mg} / \mathrm{d}$ ) estão indicadas na fase inicial, edematosa da doença e na vigência de atividade inflamatória (artrite ou miosite, por exemplo). Imunossupressores podem ser usados quando ocorre rápida progressão da doença cutânea ou de manifestações viscerais (digestivas, pulmonares, mas não as renais). As drogas mais utilizadas são o clorambucil, o metotrexato e a ciclofosfamida EV.

Como em toda doença de etiologia desconhecida e de mau prognóstico, há várias formas de tratamento sem eficiência comprovada, como vitamina E, transplante de fibroblastos e inúmeras drogas antifibróticas.

\section{DOENÇA MISTA DO TECIDO CONJUNTIVO}

Trata-se de uma síndrome de superposição de doenças auto-imunes, de características heterogêneas, na qual se somam à esclerodermia manifestações variadas de AR, DM/PM e LES. Em geral, doses moderadas de prednisona (até $40 \mathrm{mg} / \mathrm{d}$ ) controlam a grande maioria dos sintomas da doença. Quando existe predomínio de uma doença particular (por exemplo, de AR ou LES), esta é tratada como se estivesse isolada.

Como as manifestações vasculares são muito comuns (fenômeno de Raynaud ocorre em praticamente todos os casos e a hipertensão pulmonar é a principal causa de óbito), usam-se, liberalmente, vasodilatadores e, para a doença pulmonar, imunossupressores, corticoesteróides, anticoagulantes, inibidores da enzima de conversão e oxigênio inalatório.

\section{SÍNDROME DE SJÖGREN}

O tratamento das manifestações sistêmicas da síndrome de Sjögren primária, é feito, geralmente, com corticoesteróides (prednisona $1 \mathrm{mg} / \mathrm{kg} / \mathrm{d}$ ) e, nos casos refratários, drogas citotóxicas (metotrexato, azatioprina ou ciclofosfamida). 
Muitos pacientes procuram auxílio médico com seqüelas da doença, principalmente xerostomia e xeroftalmia. Elas são tratadas, respectivamente, com substitutos da lágrima (há vários preparados de lágrima artificial, alguns são irritantes e a freqüência de aplicação tem que ser ajustada à duração do efeito) e com pastas dentifrícias, géis bucais ou saliva artificial, devendo o médico estar alerta para a instalação de candidíase oral.

Na síndrome de Sjögren, secundária à $\mathrm{AR}$ ou LES, por exemplo, o tratamento é o da doença de base, associado aos substitutos da lágrima e da saliva.

\section{VASCULITES}

Constituem um grupo heterogêneo de síndromes clínicas, cujo denominador comum é a inflamação dos vasos sanguíneos. A orientação terapêutica para as vasculites dependerá da sua patogênese, da extensão do comprometimento vascular e do tempo de evolução da doença.

O tratamento das principais síndromes vasculíticas reconhecidas é discutido abaixo.

\subsection{Panarterite nodosa}

A droga mais efetiva para a terapêutica desta grave doença é a ciclofosfamida, por via oral ou, preferentemente, na forma de pulsos endovenosos, de freqüência mensal no primeiro ano, espançando-se, então, para bimestral ou trimestral. Os corticoesteróides não parecem controlar o curso da doença, particularmente renal e cerebral, mas podem ser usados para o controle das manifestações inflamatórias. Há alguns estudos, mostrando o efeito benéfico da D-penicilamina, reduzindo o tempo de uso dos imunossupressores.

A PAN limitada ou cutânea pode ser tratada com doses moderadas de prednisona e, como alternativa, sulfassalazina, danazol, dapsona e pentoxifilina.

\subsection{Granulomatose de Wegener}

A terapêutica é muito semelhante à da PAN, mas dá-se preferência à ciclofosfamida por via oral, combinada à prednisona. Após a retirada desta, a ciclofosfamida é mantida por dois anos, para evitar recidivas da doença, que são muito frequentes. Como alternativa, pode-se usar metotrexato, em pulsos semanais de $20 \mathrm{mg}$.

O envolvimento renal é o fator prognóstico mais importante, seguindo-se o pulmonar.

\subsection{Outras vasculites}

Existe um grande número de síndromes vasculíticas, a maioria das quais é tratada com corticoesteróides (prednisona $1 \mathrm{mg} / \mathrm{kg} / \mathrm{d}$ ).

$\mathrm{Na}$ vasculite secundária à crioglobulinemia, pode-se combinar plasmaferese com ciclofosfamida. As lesões vasculíticas da doença de Behscet raramente são controladas só com corticoterapia; assim, as lesões retinianas e neurológicas requerem o uso de clorambucil e as lesões pulmonares, de ciclofosfamida e prednisona. Drogas citotóxicas são também necessárias para a síndrome de Churg-Strauss.

Nas vasculites de hipersensibilidade (a drogas, agentes infecciosos, neoplasias), é importante afastar o agente desencadeante. Do mesmo modo, na vasculite hipocomplementêmica, o tabagismo deve ser interrompido, dado o papel da doença pulmonar obstrutiva crônica no prognóstico da doença.

O fenômeno de Raynaud pode ser primário (doença de Raynaud) ou secundário a doenças autoimunes, principalmente a ES e a DMTC. Em ambos os casos, utilizam-se como drogas de primeira escolha um dos bloqueadores de canais de cálcio (nifedipina, isradipina ou amlodipina), seguido da pentoxifilina, pulsos de xilocaína (ver secção 5) ou a simpatectomia.

\section{FEBRE REUMÁTICA}

\subsection{Fase aguda}

O tratamento da febre reumática aguda se faz com a erradicação do estreptococo e com a supressão dos fenômenos inflamatórios articulares e, principalmente, cardíacos.

A American Heart Society preconiza a erradicação do estreptococo com uma dose única de penicilina benzatina (600 $000 \mathrm{U}$ para pacientes $<27 \mathrm{~kg}$ e $1200000 \mathrm{U}$ para $>27 \mathrm{~kg}$ ) e, alternativamente, penicilina $\mathrm{V}$ oral por dez dias (250 $000 \mathrm{U}$ para crianças e 500 $000 \mathrm{U}$ para adultos, 3 a 4x/d). Em nosso meio, entretanto, ainda é mais usado o esquema de penicilina procaína (400 000 U IM 12/12 h por dez dias). Para os pacientes alérgicos à penicilina, usa-se a eritromicina ou um de seus novos derivados (roxitromicina, claritromicina, azitromicina), tendo sido abandonadas as sulfonamidas, por serem ineficientes nesta fase.

O esquema antiinflamatório clássico para a febre reumática aguda emprega a aspirina na dose de 80 a $120 \mathrm{mg} / \mathrm{kg}$, dividida em $6 / 6 \mathrm{~h}$, para crianças de até $30 \mathrm{~kg}$ e de 4 a $6 \mathrm{~g} / \mathrm{d}$ para adultos. Como há pouca experiência com outros AINE, nos casos refratários 
ou com manifestações cardíacas relevantes, emprega-se a prednisona $(1 \mathrm{mg} / \mathrm{kg} / \mathrm{d})$. Pulsos de metilprednisolona e outros imunossupressores têm sido utilizados em casos isolados, que não respondem à corticoterapia oral.

\subsection{Fase crônica}

Passada a fase aguda, deve-se instituir um esquema profilático para se evitar a recidiva de infecções estreptocócicas e de novos surtos de febre reumática. Usa-se, universalmente, a penicilina benzatina (1 200000 U IM) em intervalos de vinte e oito dias ou menores (vinte e um ou quatorze dias), dependendo da ocorrência de faringites comprovadas, na vigência da profilaxia. Os pacientes alérgicos à penicilina podem empregar eritromicina ou derivados, ou sulfonamidas, diariamente. A duração da cobertura antibiótica é controversa, advogando-se sua utilização, por toda a vida, para os pacientes com lesões cardíacas e até a idade adulta para os outros pacientes.

Pacientes com valvopatias crônicas ou com coréia são tratados, sintomaticamente, pelos respectivos especialistas. Recidivas de febre reumática aguda devem ser cuidadosamente diferenciadas de outras doenças (LES, AR juvenil, endocardite bacteriana) e, quando comprovadas, são tratadas como um novo surto (Secção 9.1.).

\section{GOTA E PSEUDOGOTA}

\subsection{Crise aguda}

O tratamento farmacológico da crise de gota ou pseudogota aguda se faz, atualmente, com um AINE injetável (diclofenaco, cetoprofeno, oxicams, etc) até a redução da inflamação, passando-se, então, à administração por via oral. Pode-se também associar doses baixas de colchicina $(0,5 \mathrm{mg} 1-3 \mathrm{x} / \mathrm{d})$, principalmente para os candidatos à terapêutica hipouricemiante. O esquema clássico de colchicina isolada $(0,5 \mathrm{mg} 2 / 2 \mathrm{~h}$ até a dose máxima de $8 \mathrm{mg} / \mathrm{d})$ está praticamente abandonado. Para pacientes com intolerância à colchicina e aos AINE, pode-se utilizar corticoesteróides, de preferência intra-articularmente.

Como medidas de apoio, coloca-se a articulação em repouso absoluto e aplica-se gelo no local afetado. Se o paciente estiver usando profilaxia antiuricêmica durante a crise, ela deve ser mantida; caso contrário, ela só deve ser introduzida cinco a dez dias após o término dos sintomas agudos, pelo risco de recidivas.

\subsection{Fases intercrítica e crônica}

A terapêutica antiuricêmica na fase intercrítica da gota, dependerá principalmente, da freqüência das crises agudas. Os pacientes com hiperuricemia assintomática ou com crises agudas muito espaçadas (de vários meses a anos) não têm indicação de hipouricemiantes. Estes devem ser usados quando as crises forem freqüentes ou quando a doença se cronificar. A escolha entre uma droga uricosúrica (a única disponível no Brasil é a benzopromarona, usada na dose de 50 a $100 \mathrm{mg} / \mathrm{d}$ ) e um inibidor da produção de urato (alopurinol 200-900 mg/d) depende de vários fatores. Como a grande maioria dos pacientes com gota primária tem um padrão normal de excreção de ácido úrico, na prática, pode-se utilizar tanto o alopurinol como os uricosúricos. Os uricosúricos estão contra-indicados em pacientes acima de sessenta anos, nos com aumento de creatinina ou com história prévia de cálculos renais, principalmente pelo risco de insuficiência renal. O alopurinol não é tolerado por uma proporção significativa de pacientes e não pode ser associado a droga anti-purina (azatioprina, por exemplo). A medida da excreção renal de ácido úrico está indicada apenas em pacientes jovens com suspeita de defeitos genéticos do metabolismo das purinas ou na suspeita de hiperuricemia secundária.

Nos pacientes com uma grande freqüência de crises agudas ou com artrite tofácea crônica, recomenda-se o uso contínuo de doses baixas de colchicina $(0,5$ a $2,0 \mathrm{mg} / \mathrm{d})$ como profilaxia e associa-se um hipouricemiante que deve ser o alopurinol quando houver tofos. Alimentos ricos em purina (carnes, pescados, queijos) e bebidas alcoólicas podem precipitar crises agudas e dificultar o controle da uricemia.

Já na pseudogota ou condrocalcinose, não há nenhuma intervenção que reduza significativamente a deposição articular de cristais de pirofosfato de cálcio. Em nosso serviço, temos empregado o carbonato de magnésio (50-200 $\mathrm{mg} / \mathrm{d})$, com melhora sintomática.

\section{OSTEOARTROSE}

Até a última década, a terapêutica da osteoartrose era puramente sintomática, baseada no uso de analgésicos e antiinflamatórios. Essas drogas podem ser usadas em doses mais baixas, de forma contínua ou intermitente, do que as empregadas, por exemplo, para a artrite reumatóide. Em nosso serviço, te- 
mos utilizado os antimaláricos, quando há manifestações inflamatórias proeminentes, com resultados favoráveis. Quando essas manifestações ocorrem em poucas articulações, pode-se injetar corticoesteróides de depósito (acetato de metilprednisolona, $40 \mathrm{mg} / \mathrm{ml}$ ).

Várias drogas, a maioria ainda não disponível no Brasil, têm sido usadas para estimular a formação de cartilagem articular e diminuir a atividade da doença. Entre essas, a diacereína, um inibidor da produção e liberação de IL-1, tem mostrado efeitos benéficos para osteoartrose de grandes articulações. Por outro lado, agentes biológicos, como extratos de cartilagem, não se mostraram eficientes em estudos clínicos controlados, enquanto derivados do ácido hialurônico e os sulfatos de glucosamina, de glucosaminoglicana e de condroitina mostraram benefícios.

Finalmente, é indispensável ao paciente com osteoatrtrose a correção da obesidade e de vícios posturais e a reprogramação de atividades físicas que causem estresse nas articulações acometidas. Por outro lado, exercícios físicos cuidadosamente escalonados para aumentar a força muscular para-articular e aplicação de calor ou gelo nas articulações, dependendo da preferência do paciente, também podem trazer benefícios.

\section{OSTEOPOROSE}

A terapia de reposição hormonal é o tratamento mais utilizado para a osteoporose pós-menopausa, mas ela tem que ser iniciada nos dois a quatro primeiros anos após a cessação da menstruação, pois, após esse período, a perda de massa óssea poderá não ser revertida com hormônios sexuais. Esse tratamento deverá ser feito com supervisão ginecológica, pelo risco de neoplasias. No homem, a osteoporose, associada ao hipogonadismo deve ser tratada com testosterona.

Quando a reposição hormonal for contra-indicada ou ineficiente, as alternativas terapêuticas são os bifosfonatos, as calcitoninas e a ipriflavona. Os bifosfonatos são os agentes mais eficientes da atualidade, aumentando a massa óssea tanto do fêmur como da coluna vertebral e diminuindo a frequiência de fraturas. Devem ser selecionados os compostos mais novos, como o alendronato de sódio, na dose de 10 $\mathrm{mg} / \mathrm{d}$. As calcitoninas são utilizadas desde a década de 80, possuindo grande ação analgésica sobre a fratura osteoporótica. Há vários tipos de calcitonina, mas só a de salmão é recomendada, para osteoporose vertebral, na dose mínima de $200 \mathrm{U} / \mathrm{d}$ intrananasal ou $100 \mathrm{U} / \mathrm{d}$ SC. A ipriflavona é uma nova droga disponível em nosso meio, que está sendo usada em nosso serviço, mas ainda com resultados inconclusivos.

Compostos de flúor já foram muito utilizados, pois aumentam bastante a massa óssea, predominantemente vertebral, mas o osso formado é muito frágil, não produzindo diminuição das taxas de fratura. Entretanto, nos casos de fraturas repetidas, principalmente vertebrais, refratárias ao tratamento convencional, pode-se usar compostos de fluor $(50 \mathrm{mg} / \mathrm{d}$ em ciclos de quatorze meses). Esse é também o critério para se empregar em combinações de drogas (calcitonina + estrógenos, por exemplo).

Além do tratamento específico, é necessária a complementação com sais de cálcio (1-1,5 g/d) e Vitamina D (400-800 UI/d) ou calcitriol $(0,5 \mu \mathrm{g} / \mathrm{d})$, evitar a ingestão de álcool, café, sal e fosfatos e um programa de atividades físicas.

\section{FIBROMIALGIA}

Trata-se de uma síndrome reumática dolorosa, associada a uma disfunção do metabolismo da serotonina no SNC, com alta prevalência na população adulta feminina, causando grande incapacidade funcional.

As drogas de escolha são os antidepressivos, devido a sua ação de inibir a recaptação da serotonina. Os derivados tricíclicos são os mais utilizados, nas doses correspondentes a 25 a $100 \mathrm{mg} / \mathrm{d}$ de amitriptilina. Essas drogas induzem uma grande variedade de efeitos colaterais nos primeiros dias de tratamento, desaparecendo em seguida; infelizmente, os efeitos benéficos sobre a fadiga, as dores musculares e os distúrbios do sono tendem também a diminuir ao longo do tratamento. Quando isso ocorre, pode-se usar outros tricíclicos (imipramina, clormipramina, nortriptilina, etc) ou antidepressivos não tricíclicos, como a fluoxetina e outros de ação mais seletiva sobre os receptores da serotonina, que apresentam menores efeitos colaterais, porém têm um custo mais elevado.

Nos períodos de exacerbação dolorosa, utilizamse analgésicos, AINE e miorrelaxantes. É, também, importante um programa de exercícios físicos, de preferência em água aquecida, e de apoio psicológico ou de orientação psiquiátrica.

O tratamento das doenças reumáticas sistêmicas não imunológicas está sumariado na Tabela IV. 
Tabela IV - Abordagem terapêutica das doenças reumáticas não-imunológicas

\begin{tabular}{|c|c|c|}
\hline Doença & Terapêutica farmacológica & Terapêutica não-farmacológica \\
\hline \multicolumn{3}{|l|}{ GOTA } \\
\hline - Crise aguda & $\begin{array}{l}\text { AINE IM + colchicina VO ou corticói- } \\
\text { des intra-articulares }\end{array}$ & $\begin{array}{l}\text { Repouso articular e aplicação } \\
\text { local de gelo }\end{array}$ \\
\hline - Fase intercrítica & $\begin{array}{l}\text { Alopurinol ou uricosúricos; colchicina } \\
\text { profilática }\end{array}$ & $\begin{array}{l}\text { Evitar bebidas alcoólicas e ali- } \\
\text { mentos ricos em purina }\end{array}$ \\
\hline - Fase crônica tofácea & Idem; dar preferência ao alopurinol & Idem \\
\hline \multicolumn{3}{|l|}{ PSEUDOGOTA } \\
\hline - Fase aguda & Mesmo $\mathrm{p} /$ gota aguda & Mesmo p/gota aguda \\
\hline - Fase crônica & $\begin{array}{l}\text { Colchicina profilática, carbonato de } \\
\text { magnésio }\end{array}$ & Mesmo $\mathrm{p} /$ osteoartrose \\
\hline OSTEOARTROSE & $\begin{array}{l}\text { Analgésicos ou AINE } \\
\text { Antimaláricos para inflamação contí- } \\
\text { nua e intensa }\end{array}$ & $\begin{array}{l}\text { Correção da obesidade, modifi- } \\
\text { cações da atividade diária, exer- } \\
\text { cícios, termoterapia }\end{array}$ \\
\hline OSTEOPOROSE & $\begin{array}{l}\text { Suplemento de Ca e Vit D; reposição } \\
\text { hormonal, calcitonina ou bifosfonatos }\end{array}$ & $\begin{array}{l}\text { Exercícios físicos; } \Downarrow \text { ingesta de } \\
\text { café, álcool, fosfatos, sal, } \Uparrow \mathrm{Ca}\end{array}$ \\
\hline FIBROMIALGIA & Antidepressivos & $\begin{array}{l}\text { Exercícios físicos, termoterapia, } \\
\text { suporte psicológico }\end{array}$ \\
\hline
\end{tabular}

AINE - Antiinflamatórios não-esteroidais.

PETEAN FC et al. Pharmacologic therapy of rheumatic diseases. Medicina, Ribeirão Preto, 30: 522-531, oct./dec. 1997.

ABSTRACT: We review here the current pharmacologic therapy of rheumatic diseases in adults as conducted by the Division of Clinical Immunology of the University Hospital of the School of Medicine of Ribeirão Preto of the University of São Paulo. Emphasis is posed on drug selection, clinical responses and therapeutic monitoring of leading rheumatic diseases. Among them are included those with autoimmune ethiopathogenesis (rheumatoid arthritis, systemic lupus erythematosus, rheumatic fever, spondyloarthropathies, systemic sclerosis, dermatopolymyositis and the vasculitis) and those without immunological nature (gout, pseudogout, osteoarthrosis, osteoporosis and fibromyalgia).

UNITERMS: Rheumatic Diseases. Pharmacology. Anti-Inflamatory Agents. Immunosupressive Agents.

\section{BIBLIOGRAFIA RECOMENDADA}

1 - CARVALHO IF. Drogas utilizadas na terapêutica das doenças reumáticas. Medicina (Ribeirão Preto) 28: 410-443, 1995.

2 - VOLTARELLI JC \& PETEAN FC. Emergências reumatológicas. Medicina (Ribeirão Preto) 25: 514-538, 1992.

3 - KOOPMAN WJ, ed. Arthritis and allied conditions. A textbook of rheumatology. Williams \& Wilkins, Baltimore, p. 1-2374, 1997.
4 - WEISMAN MH \& WEINBLATT ME, ed. Treatment of rheumatic diseases. Companion of the textbook of rheumatology. WB Saunders, Philadelphia, p. 1-384, 1995.

Recebido para publicação em 05/11/97.

Aprovado para publicação em 10/12/97. 